\title{
Investigation of collective effects in DIS with the ZEUS detector
}

\author{
Mariusz Przybycien ${ }^{* \dagger}$ (on behalf of the ZEUS Collaboration) \\ AGH University of Science and Technology \\ Faculty of Physics and Applied Computer Science \\ Al. Mickiewicza 30, 30-059 Cracow, Poland \\ E-mail: mariusz.przybycien@agh.edu.pl
}

\begin{abstract}
We present a preliminary measurement of two-particle correlations in collisions of electron beam at $27.5 \mathrm{GeV}$ with beam of protons at $920 \mathrm{GeV}$, which corresponds to $318 \mathrm{GeV}$ centre-of-mass energy. A sample of events equivalent to the integrated luminosity of $366 \mathrm{pb}^{-1}$ was recorded with the ZEUS experiment in 2003-2007. The correlations are measured for charged hadrons as a function of event multiplicity for the laboratory pseudorapidity range $-1.5<\eta<2$. To probe the possible contribution due to collective effects, the correlations are studied as a function of the particle's pair separation in pseudorapidity and the pair mean transverse momentum. The observed correlations are compared to available Monte Carlo models of deep inelastic electronproton scattering. Observations based on the analysis of the ZEUS data put a limit on the possible collective effects in high multiplicity electron-proton collisions.
\end{abstract}

XXXiX International Conference on High Energy Physics (ICHEP2018)

4-11 July 2018

Seoul, Korea

\footnotetext{
* Speaker.

${ }^{\dagger}$ This work was partly supported by the AGH UST statutory tasks No. 11.11.220.01/4 within subsidy of the Ministry of Science and Higher Education.
} 


\section{Introduction}

Measurements of azimuthal correlations in small collision systems, such as $p p$ or $p+\mathrm{Pb}$, in the LHC experiments, have revealed the ridge phenomenon [1, 2, 3], a feature first observed in $A+A$ collisions: enhanced production of particle pairs at small azimuthal angle separation, $\Delta \phi$, extended over a wide range of pseudorapidity separation, $\Delta \eta$. This collective particle production in $p p$ and $p+\mathrm{Pb}$ collision systems has motivated a search for similar effects in even smaller systems, such as produced in relativistic electron-proton collisions. The ridge reflects multi-parton dynamics early in the collision and has generated significant interest in the high-energy physics community. A key question is whether the long-range multi-particle collectivity reflects initial momentum correlation from gluon saturation effects [4], or a final-state hydrodynamic response to the initial collision geometry [5]. The ridge signature from the two-particle angular correlations is characterized by a Fourier decomposition of the correlation function $C(\Delta \phi) \sim 1+2 \sum_{n} \mathrm{v}_{n}^{2} \cos (n \Delta \phi)$, where $\mathrm{v}_{n}$ are the single-particle anisotropy harmonic coefficients. An important feature of the ridge is whether it involves all particles in the event (collective flow) or if it arises merely from correlations among a few particles, due to resonance decays, jets, or multi-jet production (non-flow). In small systems the contributions from non-flow sources, in particular from jets and dijets, are large. Since collective flow is intrinsically a multi-particle phenomenon, it can be probed more directly using cumulants based on multi-particle correlation techniques. The standard cumulant methods involve the calculation of $2 k$-particle azimuthal correlations $\left\langle\{2 k\}_{n}\right\rangle$, and $2 k$-particle cumulants, $\mathrm{c}_{n}\{2 k\}$, for the $n$ th-order flow harmonics. In this preliminary study presented here only 2-particle cumulants, defined as $\mathrm{c}_{n}\{2\}=\left\langle\left\langle\{2\}_{n}\right\rangle\right\rangle$, are obtained from the azimuthal correlations. The symbol $\langle\langle\rangle\rangle$ denotes averaging over both the particle pairs in an event and the event ensemble.

\section{Collectivity in DIS at HERA}

This analysis is based on $366 \mathrm{pb}^{-1}$ of data collected by the ZEUS experiment at HERA in deep inelastic scattering (DIS) process in which electrons of energy $27.5 \mathrm{GeV}$ were scattered off protons of energy $920 \mathrm{GeV}$ with a centre-of-mass energy $\sqrt{s}=318 \mathrm{GeV}$. The interaction is mediated by a highly virtual photon, with the scattered electron and the proton fragments measured in the main detector. In this analysis the scattered electrons are required to have energy $E_{e}^{\prime}>10 \mathrm{GeV}$ and the polar angle with respect to the initial electron beam direction $\theta_{e}>1 \mathrm{rad}$. The virtuality $Q^{2}$ of the exchanged photon must be greater than $5 \mathrm{GeV}^{2}$. In addition, to further suppress photoproduction, the requirement $47<\sum_{h}\left(E_{h}-p_{z, h}\right)<69 \mathrm{GeV}$ is applied, where the sum runs over all final state particles, including the scattered electron. The tracks used for obtaining cumulants are required to have transverse momentum in the range $0.1<p_{\mathrm{T}}<5 \mathrm{GeV}$ and pseudorapidity in the laboratory frame within the interval $-1.5<\eta<2$. The measured 2-particle cumulants of order $n=2,3,4$ are shown in Fig. 1 for two pair separations in pseudorapidity: $|\Delta \eta|>0$ (no separation) and $|\Delta \eta|>2$ (long range correlations). One can clearly see that all the cumulants are consistent with zero for the long range correlations. This means that we do not observe any long range collective effects in DIS. In the case of no separation, the cumulants are dominated by non-flow effects. Figure 2 shows comparison of the measured cumulant $c_{2}\{2\}$ with predictions from the two Monte Carlo models tuned to HERA data: Ariadne [6] and Lepto [7], as a function of event multiplicity, pair 

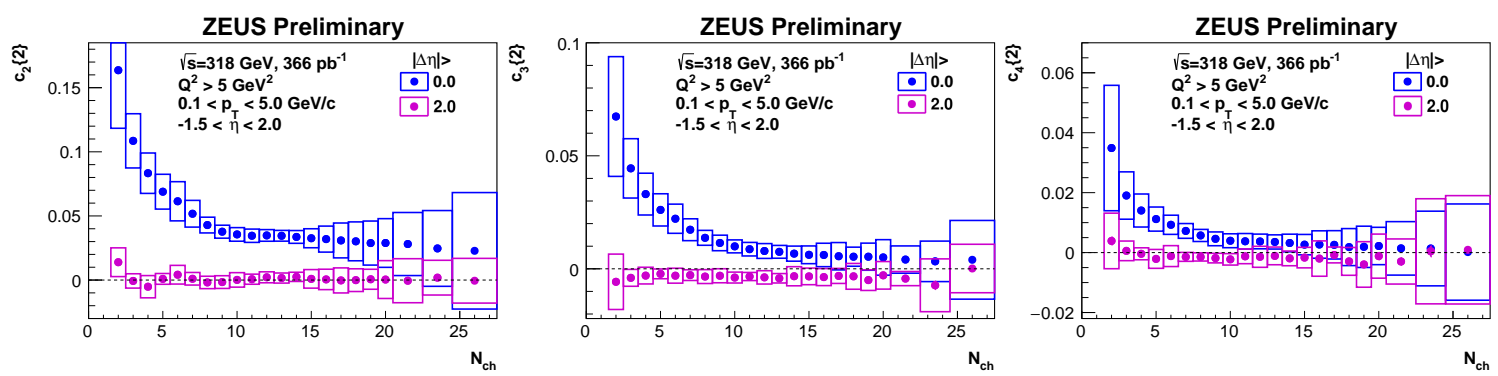

Figure 1: Two-particle cumulants of order $n=2,3,4$ (from left to right) measured in DIS events at HERA as a function of multiplicity for no $\Delta \eta$ separation between charged particles (blue) and for long range correlations $(|\Delta \eta|>2)$ (magenta). The boxes represent statistical and systematic uncertainties added in quadrature.
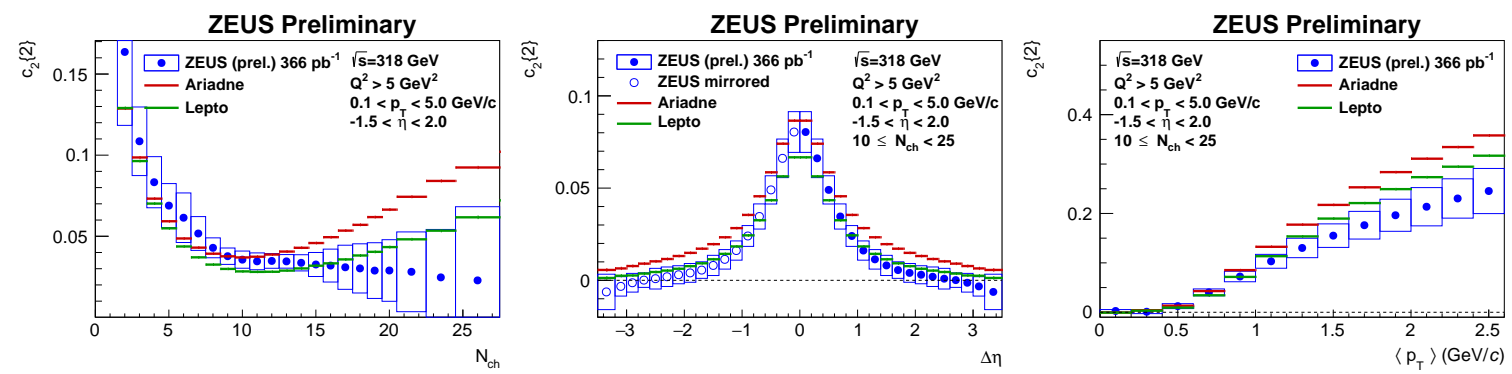

Figure 2: Comparison of the measured cumulant $c_{2}\{2\}$ with predictions from Ariadne and Lepto as a function of (left) multiplicity, (middle) psudorapidity separation of the pair, and (right) pair mean transverse momentum. The boxes represent statistical and systematic uncertainties of data points added in quadrature.

separation in pseudorapidity and mean transverse momentum of the pair. One can see that Lepto provides in each case a better description than Ariadne especially at large multiplicities.

\section{Summary}

In this preliminary search for collectivity in electron-proton DIS we did not found any signs of long-range collective effects. Although the event multiplicities available at HERA are significantly smaller than those at the LHC, we might still try to extend our analysis to more that 2-particle correlations to reduce the non-flow effects which are expected to dominate the shorter range correlations in the above measurements. We also plan to extend the analysis to photoproduction with resolved photons, as this process resembles more hadron-hadron collisions.

\section{References}

[1] CMS Collaboration, J. High Energy Phys. 09 (2010) 091, arXiv: 1009.4122 [hep-ex].

[2] ALICE Collaboration, Phys. Lett. B 719 (2013) 29, arXiv: 1212.2001 [nucl-ex].

[3] ATLAS Collaboration, Phys. Rev. Lett. 110 (2013) 182302, arXiv: 1212.5198 [hep-ex].

[4] K. Dusling, R. Venugopalan, Phys. Rev. D 87 (2013) 094034, arXiv: 1302.7018 [hep-ph].

[5] P. Bozek, W. Broniowski, Phys. Rev. C 88 (2013) 014903, arXiv: 1304.3044 [nucl-th].

[6] L. Lönnblad, Comput. Phys. Commun. 71 (1992) 15.

[7] G. Ingelman, A. Edin, J. Rathsman, Comput. Phys. Commun. 101 (1997) 108, arXiv: hep-ph/9605286. 